\title{
Major life events, stress appraisal, and migraine: results of the American Migraine Prevalence and Prevention (AMPP) study
}

\author{
AN Manack ${ }^{*}$, D Serrano ${ }^{2}$, CC Turkel ${ }^{3}$, RB Lipton ${ }^{4}$, DC Buse ${ }^{4}$ \\ From The European Headache and Migraine Trust International Congress \\ London, UK. 20-23 September 2012
}

\section{Objective}

To assess cross-sectional differences among persons with chronic migraine (CM) versus episodic migraine (EM) in major-life-events (MLE) rates and appraisal of events as stressful (SLE).

\section{Methods}

AMPP is a longitudinal, US-population-based study for which questionnaires were mailed to 24,000 severe headache sufferers and followed annually. Respondents with ICHD-2 migraine were stratified as either CM ( $\mathrm{i} Y 15$ headache-days/month) or EM ( $<15$ headache-days/month). MLE occurrences were defined as moving, change in significant-relationship status, work/school stressors, events w/children, deaths, other over preceding year. For endorsed MLEs, respondents were asked to assess stress level on a 5 -point Likert scale $\left(1=i^{\circ}\right.$ not at all $i_{i} \pm / 5=i^{0}$ very \pm$)$. To identify a SLE, responses were dichotomized with a cut-score of $; Y$ Y 4 . Ordered logistic regressions used to model odds of reporting more SLEs. Results In 2007, 14,069 individuals responded and 557 had CM and 748 had EM. $80.1 \%$ of CM reported $i Y ́ 1$ MLE in preceding year vs $78.6 \%$ of EM. The proportion of CM vs EM reporting no MLEs (18.2 vs $21.4 \%$ ), 1 MLE (26.3 vs $27.5 \%$ ), 2 MLEs (27.8 vs $25.1 \%$ ) or ¡ $\hat{Y} 3$ MLEs (27.8 vs $25.1 \%$ ) revealed more MLEs for CM. $76.5 \%$ of CM reported iÝ1 SLE in preceding year vs $71.4 \%$ of EM. Proportion of CM vs EM reporting no SLEs (19.3 vs 23.3\%), 1 SLE (32.9 vs $34.1 \%), 2$ SLEs (27.2 vs $25.2 \%$ ) or ¡Ý3 SLEs (20.7 vs $17.4 \%)$ revealed more SLEs for CM. Unadjusted odds ratio (OR) comparing those with stress scores $4 \& 5 \mathrm{w} /$ lower scores was $\sim 25 \%$ greater for CM vs EM (OR=1.25, 95\%CI 1.041.49). Adjusting for age, gender and race produced similar.

\section{Results}

(OR=1.27, 95\%CI 1.07-1.52).

\section{Discussion}

CM persons reported more MLEs in the preceding year and were more likely to perceive events as stressful. Longitudinal analyses are required to assess whether MLEs/SLEs are risk factors for"Cor consequences of ${ }^{\circ C M}$.

\section{Funding}

The AMPP study was funded through a research grant to the NHF from Ortho-McNeil Neurologics. Additional analyses were supported by Allergan, Inc.

\section{Author details}

${ }^{1}$ Allergan, Inc., Irvine, California, USA. ${ }^{2}$ Vedanta Research, Chapel Hill, North Carolina, USA. ${ }^{3}$ Allergan, Inc, USA. ${ }^{4}$ Department of Neurology, Albert Einstein College of Medicine and the Montefiore Headache Center, Bronx, New York, USA.

Published: 21 February 2013

doi:10.1186/1129-2377-14-S1-P9

Cite this article as: Manack et al:: Major life events, stress appraisal, and migraine: results of the American Migraine Prevalence and Prevention (AMPP) study. The Journal of Headache and Pain 2013 14(Suppl 1):P9. 